$\rho(r)=\xi(r)$ for $t_{2} \leqq r \leqq s_{2}$ where $t_{2}\left(<s_{2}\right)$ is the point nearest to $s_{2}$ at which $\xi\left(t_{2}\right)=\sigma\left(t_{2}\right)$. If $\xi\left(s_{2}\right)=\sigma\left(s_{2}\right)$, then let $t_{2}=s_{2}$. For $r<t_{2}$ let $\rho(r)$ $=\rho\left(t_{2}\right)+\log \log \log t_{2}-\log \log \log r$ for $u_{1} \leqq r \leqq t_{2}$ where $u_{1}\left(<t_{2}\right)$ is the point of intersection of $y=\rho$ with $y=\rho\left(t_{2}\right)+\log \log \log t_{2}-\log \log \log r$.

Let $\rho(r)=\rho$ for $r_{1} \leqq r \leqq u_{1}$. It is always possible to choose $r_{2}$ so large that $r_{1}<u_{1}$. We repeat the procedure and note that

$$
\rho(r) \geqq \xi(r) \geqq \sigma(r)
$$

and $\rho(r)=\sigma(r)$ for $r=t_{1}, t_{2}, t_{3}, \cdots$. Hence $\lim _{r \rightarrow \infty} \rho(r)=\rho$, and

$$
\limsup _{r \rightarrow \infty} \frac{\log M(r)}{r^{\rho(r)}}=1 \text {. }
$$

MUSLIM UNIVERSITY

\title{
A NOTE ON THE SPECTRAL THEOREM
}

\section{WILLIAM F. EBERLEIN}

1. Introduction. Although the connections between the spectral resolution of a self-adjoint transformation in Hilbert space, the moment problem, and Riesz' integral representation [1] ${ }^{1}$ for linear functionals on the space $C$ are known (cf. Stone [2], Murray [3], Widder [4], Lengyel [5]), the following elementary derivation of the spectral theorem from the Riesz theorem exhibits the connections in, perhaps, the simplest light. We consider only bounded self-adjoint transformations $H$; one can treat an unbounded $H$ by considering $\left(I+H^{2}\right)^{-1}$, which is bounded and self-adjoint $[3$, p. 95]. Note that the derivation does not involve the separability of the Hilbert space $\mathfrak{W}$.

2. Six lemmas. Let $H$ be a self-adjoint transformation with the bounds $a, b$ - that is, $a\|f\|^{2} \leqq(H f, f) \leqq b\|f\|^{2}$ for all $f \in H$, and $\|H\|$ $=\max (|a|,|b|)$. Denote by $C$ the space of continuous real-valued functions defined on the closed interval $(a, b)$, with $\|f(x)\|=\max |f(x)|$ $(a \leqq x \leqq b)$. Let $p(x)=\sum_{0}^{n} c_{j} x^{i}$ be any polynomial with real coefficients, and let $p(H)$ be the corresponding transformation $p(H)=\sum_{0}^{n} c_{j} H^{i}$, where $H^{0}=I$.

Received by the editors September 6, 1945.

${ }^{1}$ Numbers in brackets refer to the references cited at the end of the paper. 


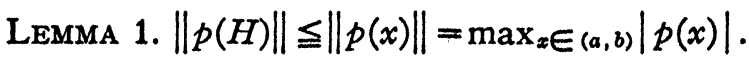

Murray [3, p. 82] gives a proof of the lemma; we remark only that the general case can be reduced to the case of a self-adjoint $H$ in $n$-dimensional Euclidean space. The inequality is almost obvious when $H$ is represented by a finite diagonal matrix; and the lemma is essentially equivalent to the statement that every $n^{2}$-symmetric matrix can be reduced to diagonal form by unitary transformations.

Given any two elements $f, g \in \mathfrak{E}$, consider the expression $(p(H) f, g)$, taking on real or complex values, as an operator defined over the linear set of polynomials included in $C$. The linearity of the operator is obvious; its continuity follows from Lemma 1:

$$
|(p(H) f, g)| \leqq\|p(H)\| \cdot\|f\| \cdot\|g\| \leqq\{\|f\| \cdot\|g\|\}\|p(x)\| .
$$

Since the polynomials are dense in $C$ (Weierstrass approximation theorem), the operator can be extended uniquely to a linear functional defined over all $C$, without increase of norm. The Riesz theorem [1] then yields immediately:

LEMMA 2. There exists a function of bounded variation $\rho(\lambda ; f, g)$ $(a \leqq \lambda \leqq b)$ such that

$$
(p(H) f, g)=\int_{a}^{b} p(\lambda) d \rho(\lambda ; f, g),
$$

the uniqueness of $\rho$ being assured by the normalization conditions: $\rho(a ; f, g)=0$ and $\rho(\lambda ; f, g)=\rho(\lambda+0 ; f, g), a<\lambda<b . \rho(\lambda ; f, f)$ is real, and $\operatorname{Var}_{(a, b)} \rho(\lambda ; f, f) \leqq\|f\|^{2}$.

The structure of the $\rho(\lambda ; f, g)$ is implied by the following elementary properties of the Stieltjes integral (cf. Widder [4]).

LEMMA 3. Let $f(x), g(x)$ be continuous and $\gamma(x)$ be a normalized function of bounded variation on $(a, b)$. Then $G(x)=\int_{a}^{x} g(t) d \gamma(t)$ is a normalized function of bounded variation, and $\int_{a}^{b} f(t) d G(t)=\int_{a}^{b} f(t) g(t) d \gamma(t)$. If $\int_{a}^{b} t^{n} d \gamma(t)=0(n=0,1,2, \cdots)$, then $\gamma(t) \equiv 0$.

LEMMA 4. (1) $\rho(b ; f, g)=(f, g), 0 \leqq \rho(\lambda ; f, f) \leqq\|f\|^{2}$;

(2) $\rho\left(\lambda ; f_{1}+f_{2}, g\right)=\rho\left(\lambda ; f_{1}, g\right)+\rho\left(\lambda ; f_{2}, g\right)$;

(3) $\rho(\lambda ; c f, g)=c \rho(\lambda ; f, g)$;

(4) $\rho(\lambda ; f, g)=\bar{\rho}(\lambda ; g, f)$.

The equality in (1) arises on setting $p(\lambda)=1$ in Lemma 2 . But then $\rho(\lambda ; f, f)$ is nondecreasing, whence the inequality. The proof of (2) is typical of the remaining statements: 


$$
\begin{aligned}
\int_{a}^{b} \lambda^{n} d \rho\left(\lambda ; f_{1}+f_{2}, g\right) & =\left(H^{n}\left(f_{1}+f_{2}\right), g\right)=\left(H^{n} f_{1}, g\right)+\left(H^{n} f_{2}, g\right) \\
& =\int_{a}^{b} \lambda^{n} d \rho\left(\lambda ; f_{1}, g\right)+\int_{a}^{b} \lambda^{n} d \rho\left(\lambda ; f_{2}, g\right) \\
& =\int_{a}^{b} \lambda^{n} d\left\{\rho\left(\lambda ; f_{1}, g\right)+\rho\left(\lambda ; f_{2}, g\right)\right\} .
\end{aligned}
$$

Set $\gamma(\lambda)=\rho\left(\lambda ; f_{1}+f_{2}, g\right)-\left\{\rho\left(\lambda ; f_{1}, g\right)+\rho\left(\lambda ; f_{2}, g\right)\right\}$ and apply Lemma 3 .

Lemma 4 and the representation theorem [2, p. 63] for bounded, bilinear symmetric functionals defined over a Hilbert (or unitary) space yield the following theorem:

LEMma 5. There exists a set of bounded self-adjoint transformations $F(\lambda)(a \leqq \lambda \leqq b)$ such that $(F(\lambda) f, g)=\rho(\lambda ; f, g) . F(a)=0$, and $F(b)=I$.

That the $F(\lambda)$ are projections is a consequence of the following result:

Lemma 6. $F(\mu) F(\lambda)=F(\nu)$, where $\nu=\min (\mu, \lambda)$. In particular, $F^{2}(\lambda)=F(\lambda)$.

For, let $m$ and $n$ be arbitrary $(m, n=0,1,2, \cdots)$. Then

$$
\begin{aligned}
\int_{a}^{b} \lambda^{n} d\left\{\int_{a}^{\lambda} \mu^{m} d(F(\mu) f, g)\right\} & =\int_{a}^{b} \lambda^{n} \cdot \lambda^{m} d(F(\lambda) f, g)=\left(H^{n+m} f, g\right) \\
& =\left(H^{n} f, H^{m} g\right)=\int_{a}^{b} \lambda^{n} d\left(F(\lambda) f, B^{m} g\right) \\
& =\int_{a}^{b} \lambda^{n} d\left(H^{m} F(\lambda) f, g\right) \\
& =\int_{a}^{b} \lambda^{n} d\left\{\int_{a}^{b} \mu^{m} d(F(\mu) F(\lambda) f, g)\right\} .
\end{aligned}
$$

Application of Lemma 3 yields

$$
\int_{a}^{b} \mu^{m} d(F(\mu) F(\lambda) f, g)=\int_{a}^{\lambda} \mu^{m} d(F(\mu) f, g)=\int_{a}^{b} \mu^{m} d(F(\nu) f, g) ;
$$

and another application, $F(\mu) F(\lambda)=F(\nu)$, where $\nu=\min (\mu, \lambda)$.

3. The spectral theorem. In order to put our results into standard form we modify the definition of the family of projections by setting 
$E(\lambda)=0(\lambda<a), E(\lambda)=F(\lambda+0)(a \leqq \lambda<b), E(\lambda)=I(\lambda \geqq b)$. The $E(\lambda)$ thus obtained form a finite resolution of the identity: They are all projections; $E(\lambda) E(\mu)=E(\nu)$, where $\nu=\min (\mu, \lambda) ; E(\lambda+0)=E(\lambda)$ $(-\infty<\lambda<\infty) ;(E(\lambda) f, g)$ is of bounded variation; $E(\lambda)=0(\lambda<a)$ and $E(\lambda)=I(\lambda \geqq b)$.

To allow for the change in definition at the point $\lambda=a$ we modify the limits of integration from $(a, b)$ to $(a-\epsilon, b), \epsilon$ being an arbitrary positive number. Our final result is then the following:

Spectral Theorem. Let $H$ be a bounded self-adjoint transformation with bounds $a, b$. Then there exists a finite resolution of the identity $E(\lambda)$ such that

$$
(p(H) f, g)=\int_{a-c}^{b} p(\lambda) d(E(\lambda) f, g),
$$

$\epsilon$ being an arbitrary positive number.

\section{REFERENCES}

1. F. Riesz, Sur les opérations functionelles linéaires, C. R. Acad. Sci. Paris vol. 149 (1909) pp. 974-977.

2. M. H. Stone, Linear transformations in Hilbert space, Amer. Math. Soc. Colloquium Publications, vol. 15, 1932.

3. F. J. Murray, An introduction to linear transformations in Hilbert space, 1941.

4. D. V. Widder, The Laplace transformation, 1941.

5. B. A. Lengyel, Bounded self-adjoint operators and the problem of moments, Bull. Amer. Math. Soc. vol. 45 (1939) pp. 303-306.

Washington, D. C. 